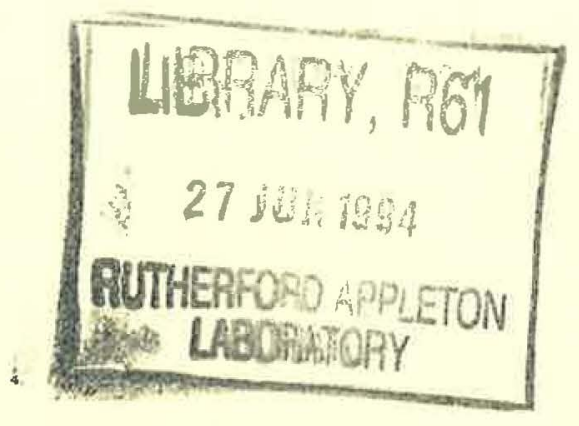

\title{
Factorization in Closed String Field Theory
}

F Anton A Abdurrahman and $\mathrm{J}$ Bordes

June 1994 
DRAL is part of the Engineering and Physical

\section{Sciences Research Council}

The Engineering and Physical Sciences Research Council does not accept any responsibility for loss or damage arising from the use of information contained in any of its reports or

in any communication about its tests or investigations 


\title{
FACTORIZATION IN CLOSED STRING FIELD THEORY
}

\author{
F. Anton*, A. Abdurrahman ${ }^{\dagger}, J$. Bordes $^{\dagger}$ \\ Department of Particle Physics \\ Rutherford Appleton Laboratory \\ DIDCOT, Oxon OX11 OQX \\ $U . K$.
}

\begin{abstract}
The so long made assumption, that a general closed-string vertex opereator $V$ should be built as a product of left- and right-moving vertex operators, rests on the fact that closed-string Fock space is constructed as a tensor product of left- and right-moving open-string Fock spaces. In this letter we will relax this assumption by proving that factorization of closed-string vertices is a general rule.
\end{abstract}

March 1994

${ }^{*}$ Dept. of Theoretical Physics, University of Oxford, 1 Keble Road, Oxford OX1 3N, U.K.

†E-Mail: abdurrahman@v2.rl.ac.uk

†Departament de Física Teórica, Universitat de Valencia, Dr. Moliner 50, E-46100, Burjassot, Spain and IFIC, Centro Mixto Universitat de Valencia-CSIC, Spain. 
The problem of formulating a covariant closed string field theory proved to be surprisingly difficult. A straightforward extension of Witten's open string field theory [1] to closed strings does not satisfy gauge invariance[2] and fails to reproduce the correct dual scattering amplitudes $[3,4]$.

It is now well established that a consistent closed string field theory, is necessarily nonpolynomial[5]. At the classical level, the interaction terms are the so-called restricted polyhedra $[6,7,8]$, where the contact interactions are given by the patterns of string overlaps on the surface of a sphere having always three edges at each vertex. For the $\mathrm{N}$-string scattering one has an $\mathrm{N}$-faced polyhedron for which the $\mathrm{N}$ closed strings correspond to the faces, and are glued together across the edges. The lengths of the edges play the role of the modular parameters. They are restricted by: (i), the perimeter of any face $F$ equals $2 \pi$ (corresponding to the fact that all strings are taken to have the same $\sigma$-length equal to $2 \pi$ ), and (ii), any closed path surrounding two or more faces on a polyhedron has length greater than or equal to $2 \pi$.

On the other hand, motivated by the similarities between Yang-Mills theory and Witten's open string field theory, it was first suggested in $[9,10]$ and proved rigorously in $[11,12,13]$, that physical open strings can be viewed as infinite dimensional matrices. If one breaks the open string into two pieces, the string field $\Psi$ can be treated as a functional of the two halves of the string, which play the role of the row and column indices, and a function of the mid-point. In particular the open-string 3 -vertex can be represented as a trace[11,12,13]. This trace can be generalized to represent any $\mathrm{N}$-string $(N \geq 3)$ tree level scattering amplitude[15]:

$$
A_{N}=\int_{-\infty}^{\infty} \frac{d \lambda_{1} \cdots d \lambda_{N}}{S L(2, \Re)} \operatorname{Tr}\left(\exp \left(\lambda_{1} M\right) A_{1} \cdots \exp \left(\lambda_{N} M\right) A_{N}\right) .
$$

The operator $M$ is the generator of infinitesimal shifts of the mid-point of the string, so in fact one is shifting this point to every possible position.

In an analogous construction for closed strings one breaks the closed-string into two pieces, therefore singling out two points, and treats each of the remaining halves as labeling matrix indices. Shifting these two points around corresponds to varying the lengths of the basic overlaps of the strings. In other words, one is varying the lengths of the edges of the polyhedra. It has been proved, Using a functional approach, in [15], that the analogous of eq. (.1) for closed strings gives the correct dual amplitudes. One could restrict the region of integration so that the restricted polyhedra are obtained. For the 3 -vertex there 
are no modular parameters, so the Witten vertex should work. For higher vertices the proper region of integration has to be determined by the requirement that the moduli spaces of the field theory are covered correctly. The organization of the paper is as follows.

We first present an oscillator construction for the orbital degrees of freedom of the closed-string based on half-string formulation (the ghost degrees of freedom will be treated in a different paper). Next we calculate explicitly the 1,2 and 3 -vertices and address the problem of factorization. The calculation of closed string 3-amplitudes and the proof that a vertex with no operator insertions obtained by sewing two vertices, satisfies the "gluing and resmoothing theorem" of [18] is presented in [19]. The problem of how the moduli space is covered for higher order amplitudes in the half-string formulation has been discussed in another paper [16]. Finally our conclusions are presented. Our approach will follow closely the approach of references $[11,14,12]$ and [13].

The Half-String Coordinates. The boundary condition satisfied by the closed string is (the space-time index suppressed, and the length of the string is taken to be $\pi), \quad X(\sigma, \tau)=X(\sigma+\pi, \tau)$, has the standard solution, at $\tau=0$,

$$
X(\sigma, 0)=x_{0}+\frac{1}{\sqrt{2}} \sum_{n \geq 1}\left[x_{n} \cos 2 n \sigma+y_{n} \sin 2 n \sigma\right],
$$

where

$$
\begin{aligned}
& x_{n}=\frac{i}{\sqrt{2} n}\left(\alpha_{n}-\alpha_{-n}+\tilde{\alpha}_{n}-\tilde{\alpha}_{-n}\right)=x_{n}^{\dagger}, \\
& y_{n}=\frac{1}{\sqrt{2} n}\left(-\alpha_{n}-\alpha_{-n}+\tilde{\alpha}_{n}+\tilde{\alpha}_{-n}\right)=y_{n}^{\dagger} .
\end{aligned}
$$

The oscillator modes $\alpha$ and $\tilde{\alpha}$, satisfy the well known commutation relations $\left[\alpha_{n}, \alpha_{m}\right]=\left[\tilde{\alpha}_{n}, \tilde{\alpha}_{m}\right]=m \delta_{n+m} ;\left[\alpha_{n}, \tilde{\alpha}_{m}\right]=0$. The Fock space of the theory is constructed by acting on the vacuum $|0\rangle$, with the creation operators $\alpha_{-n}$ and $\tilde{\alpha}_{-n},($ with $n \geq 1)$.

There are several possible boundary conditions for the half string coordinates. The correct choice of boundary conditions is the one that isolates completely the motion of the two points $X(\sigma=0)$ and $X\left(\sigma=\frac{\pi}{2}\right)$, where we break the string. This is so that there is a one-to-one correspondence between the two descriptions (which is given by an non-singular infinite dimensional matrix). We define the half-string coordinates as

$$
\chi^{1}(\sigma, \tau)=X(\sigma, \tau)-X(0, \tau)
$$




$$
\begin{aligned}
\chi^{2}(\sigma, \tau) & =X(\pi-\sigma, \tau)-X(0, \tau) \\
X_{I} & =\frac{1}{2}\left(X(0, \tau)+X\left(\frac{\pi}{2}, \tau\right)\right),
\end{aligned}
$$

where $\sigma \in\left[0, \frac{\pi}{2}\right)$. The set of equations proposed contain all the string degrees of freedom; this will become apparent once the relation between the oscillator modes in both representations is worked out. On the other hand if we add, as one naively would, the combination orthogonal to $X_{I}$, the change of representation between the full-string and the half-string degrees of freedom becomes singular. ${ }^{3}$

We will see that, although we have singled out two preferred points in the string, the theory remains local since only the plus combination, $X_{I}$, is relevant. Finally one can easily check that the overlap equations of two half strings are completely recovered in this representation.

Now, taking an odd expansion of $\chi^{r}(\sigma, \tau)$ to the interval $\sigma \in\left(-\frac{\pi}{2}, 0\right]$ we have a Fourier decomposition (at $\tau=0$ ) in terms of even sine modes only

$$
\chi^{r}(\sigma)=\sum_{n \geq 1} \chi_{n}^{r} \sin 2 n \sigma,
$$

which converges pointwise to $\chi^{r}(\sigma)$ in the open interval $\sigma \epsilon\left(-\frac{\pi}{2}, \frac{\pi}{2}\right)$. The inverse Fourier transform is

$$
\chi_{n}^{r}=\frac{4}{\pi} \int_{0}^{\frac{\pi}{2}} d \sigma \chi^{r}(\sigma) \sin 2 n \sigma .
$$

Let us define the following quantities in the space of "even-odd oscillators"

$$
\mathbf{A}_{n}^{r}=\left(\begin{array}{c}
A_{2 n}^{r} \\
A_{2 n-1}^{r}
\end{array}\right) ; \quad \mathbf{B}_{n}=\left(\begin{array}{c}
B_{2 n} \\
B_{2 n-1}
\end{array}\right),
$$

Where $\mathbf{A}(\mathbf{B})$ is any half-string (full-string) vector (bold-faced quantities will be used to represent vectors or matrices in this "even-odd" space). Then one can express $\chi_{n}^{r}$ as

$$
\chi_{n}^{r}=-\sqrt{2} \sum_{m \geq 1} \mathbf{B}_{n, m} \mathbf{u x}_{m}+\frac{(-)^{r+1}}{\sqrt{2}} \mathbf{y}_{n} ; \quad \mathbf{u}=\left(\begin{array}{cc}
0 & 1 \\
1 & 0
\end{array}\right),
$$

where

$$
\mathbf{B}_{n, m}=\left(\begin{array}{cc}
\left(\frac{2 n}{2 m-1}\right) B_{2 n, 2 m-1} & 0 \\
0 & \left(\frac{2 m}{2 n-1}\right) B_{2 n-1,2 m}
\end{array}\right),
$$

with

$$
B_{m, n}=\frac{1}{\pi}\left(\frac{2 n}{n^{2}-m^{2}}\right) .
$$

\footnotetext{
${ }^{3}$ This is because the inclusion of the orthogonal combination to $X_{I}$ results in constraints on the half-string degrees of freedom.
} 
The matrix $\mathbf{B}_{n, m}$ is non-singular ${ }^{4}$. Indeed it can be checked that:

$$
\left(\mathbf{B}^{-1}\right)_{m, n}=-4\left(\begin{array}{cc}
B_{2 m-1,2 n} & 0 \\
0 & B_{2 m, 2 n-1}
\end{array}\right) .
$$

The inverse of equation (.2) reads

$$
\mathbf{x}_{m}=\frac{-\mathbf{u}}{2 \sqrt{2}} \sum_{n, r}\left(\mathbf{B}^{-1}\right)_{m, n} \boldsymbol{\chi}_{n}^{r} ; \quad \mathbf{y}_{n}=\frac{1}{\sqrt{2}} \sum_{r=1,2}(-)^{r+1} \chi_{n}^{r} .
$$

To complete the set of the full-string oscillator modes, we still need the expression for the center of mass coordinate $x_{0}$. It is easy to see that

$$
x_{0}=X_{I}+\frac{1}{\pi} \sum_{n, r} \frac{\chi_{2 n-1}^{r}}{2 n-1} .
$$

Hence there exists a one-to-one mapping of the half-string oscillator modes to the full-string ones. To establish this one-to-one mapping at the level of Fock spaces, we need to quantize the theory.

The quantization program can be carried out in the usual way by interpreting the oscillator modes $\chi_{n}^{r}$ and $X_{I}$ as q-operators and define their conjugate momenta as $P_{I}=-i \frac{\partial}{\partial X_{I}}$, and $P_{n}^{r}=-i \frac{\partial}{\partial \chi_{n}^{r}}$. They satisfy the usual commutation relations $\left[\chi_{n}^{r}, P_{n}^{s}\right]=i \delta^{r, s} \delta_{n, m},\left[X_{I}, P_{I}\right]=i$. The corresponding momenta for the full-string are given by $P_{n}=-i \frac{\partial}{\partial x_{n}}, \tilde{P}_{n}=-i \frac{\partial}{\partial y_{n}}$ and $P=-i \frac{\partial}{\partial x_{0}}$.

The transformation between the half-string and the full-string momenta can be easily worked out using the chain rule giving

$$
\mathbf{P}_{m}^{r}=-\frac{\mathbf{u}}{2 \sqrt{2}} \sum_{n \geq 1}\left(\mathbf{B}^{-1}\right)_{n, m} \mathbf{P}_{n}+\frac{(-)^{r+1}}{\sqrt{2}} \tilde{\mathbf{P}}_{m}+\frac{\mathbf{e}_{2}}{\pi} \frac{P}{(2 m-1)}
$$

and

$$
P=P_{I}
$$

Here $\mathbf{e}_{2}$ is the unit vector $(0,1)^{T}$. These relations can be inverted to solve for $P_{m}$

$$
\mathbf{u} \mathbf{P}_{m}=-\sqrt{2} \sum_{n, r} \mathbf{B}_{n, m} \mathbf{P}_{n}^{r}+\frac{\mathbf{e}_{2}}{\sqrt{2}} P \quad \text { and } \quad \tilde{\mathbf{P}}_{m}=\sum_{r} \frac{(-)^{r+1}}{\sqrt{2}} \mathbf{P}_{m}^{r} .
$$

$P$ is recovered by multiplying the first eq. in (.5) by $\frac{1}{2 k-1}$, and summing over $r=1,2$ and odd $n \geq 1$. Doing so we arrive at

$$
P=\frac{4}{\pi} \sum_{r, k} \frac{1}{2 k-1} P_{2 k-1}^{r}
$$

\footnotetext{
${ }^{4}$ The matrix $B_{n m}$ is bounded $(|B| \leq 1)$ and its range is the whole Hilbert space $l^{2}$, hence it has an inverse.
} 
Let us pause for a moment to see the meaning of this. Traditionally, people have been bias against using the half-string approach to describe closed strings because this would produce a bilocal theory as a result of singling out two points of the string. However from (.3) and (.4) we see that the string fields depend on the two preferred points only through the combination $X_{I}$.

The full-string Fock space is build up by acting on the vacuum $|0\rangle$ with the creation operators

$$
a_{m}^{\dagger}=\frac{1}{\sqrt{2 m}}\left(\alpha_{-m}+\tilde{\alpha}_{-m}\right) ; \quad \tilde{a}_{m}^{\dagger}=\frac{i}{\sqrt{2 m}}\left(\alpha_{-m}-\tilde{\alpha}_{-m}\right) .
$$

The creation and annihilation operators for the half-string defined as

$$
\beta_{n}^{r \dagger}=i \frac{\sqrt{n}}{2} \chi_{n}^{r}+\frac{1}{\sqrt{n}} P_{n}^{r} ; \quad \beta_{n}^{r}=-i \frac{\sqrt{n}}{2} \chi_{n}^{r}+\frac{1}{\sqrt{n}} P_{n}^{r},
$$

which are related to the full string oscillator modes by

$$
\mathbf{u} \mathbf{a}_{m}=\sum_{n \geq 1}\left(\mathbf{A}_{m n}^{(+)} \mathbf{b}_{n}^{(+) \dagger}+\mathbf{A}_{m n}^{(-)} \mathbf{b}_{n}^{(+)}\right)+\frac{\mathbf{e}_{2} P}{2 \sqrt{m}} ; \text { and } \tilde{\mathbf{a}}_{m}=\mathbf{b}_{m}^{(-)}
$$

where $b_{n}^{ \pm}=\frac{1}{\sqrt{2}}\left(\beta_{n}^{(1)} \pm \beta_{n}^{(2)}\right)$. The matrix $A_{ \pm}$is given by

$$
\mathbf{A}_{m, n}^{ \pm}=\left(\begin{array}{cc}
M_{n, m}^{ \pm} & 0 \\
0 & -M_{m, n}^{ \pm}
\end{array}\right)
$$

where

$$
M_{m n}^{ \pm}=\left(\frac{2 m}{2 n-1}\right)^{\frac{1}{2}}\left[B_{2 n-1,2 m} \pm B_{2 m, 2 n-1}\right]
$$

Notice that the matrix $M^{ \pm}$has already appeared in $[11,14]$ for the open bosonic string. We see from these relations that the $a_{n}$ oscillators, for $n$ : even, odd, separately behave as though they were two independent open strings as far as the change of representation is concerned. To make the connection with the half-string formulation we need to relate the full string vacuum $\mid 0>$ to the half-string vacua $\mid 0>_{1}$ and $\mid 0>_{2}$ corresponding to the two halves of the string. The half-string vacua are defined through the relations $\beta_{m}^{r} \mid 0>_{r}=0$. Repeated action of the creation operators $\beta_{m}^{r \dagger}$, on these vacua gives the entire Fock spaces corresponding to each half-string. We propose the standard ansatz

$$
\left|0>=\exp \left(\frac{-1}{2} \mathbf{b}_{n}^{(+) \dagger} \boldsymbol{\xi}_{n m} \mathbf{b}_{m}^{(+) \dagger}\right)\right| 0>_{1} \mid 0>_{2} .
$$


Notice that only the plus combination appears in the exponential. This is because $\tilde{a}_{n}$ is expressed only in terms of $b_{n}^{(-)}$, and hence it annihilates the vacuum trivially. The action of $a_{n}$ fixes $\boldsymbol{\xi}$ to be

$$
\boldsymbol{\xi}=\mathbf{A}^{(-)-1} \mathbf{A}^{(+)} \text {. }
$$

The tachyon state is obtained by inserting the factor $e^{i P x_{0}}$ in (.8). One can verify that this state in the half-string representation is an eigenstate of the momentum operator with an eigenvalue $P$, when expressed in the half-string language.

The Hilbert space $\mathcal{H}$ of the closed full-string is spanned by linear combinations of the state vectors of the form $\Pi a_{n}^{\dagger}, \tilde{a}_{n}^{\dagger} \mid 0>$. The one-to-one mapping between the full and half-string modes, together with (.8) imply that the Hilbert space of the full-string is contained in the completion of the tensor product $\overline{\mathcal{H}}=\overline{\mathcal{H}_{1} \otimes \mathcal{H}_{2} \otimes \mathcal{H}_{M}}$.

Matrix Representations and Vertices. To construct the half-string matrix representing a string state with arbitrary occupation numbers, it is useful to introduce the coherent state

$$
\mid \vec{\lambda}, \overrightarrow{\tilde{\lambda}} ; p)=e^{i p x_{0}} \exp \left(\lambda \cdot a^{\dagger}+\tilde{\lambda} \cdot \tilde{a}^{\dagger}\right) \mid 0>.
$$

Also one introduces the half-string states

$$
\left|n_{i}^{r}>=\prod_{i=1}^{\infty} \frac{1}{{\sqrt{n_{i}^{r}}}^{r}}\left(\beta_{i}^{r}\right)^{\dagger n_{i}^{r}}\right| 0>_{r},
$$

Now the matrix representing the functional of any state is defined by

$$
\left.[A]_{n_{i}^{1}}^{n_{2}^{2}}=(-)^{\sum_{i \geq 1} n_{i}^{2}}<n_{i}^{1} ; n_{i}^{2} \mid \lambda, \bar{\lambda} ; p\right)
$$

The factor $(-)^{\sum_{i \geq 1} n_{i}^{2}}$ takes into account that the orientation for $\chi^{2}$ is reversed.

After a long calculation we find the following expression for the matrix in $(.10)$

$$
\begin{aligned}
{[A]_{n_{i}^{1}}^{n_{i}^{2}}=} & C(\lambda ; p) e^{\frac{i p}{2} X_{I}} \prod_{i=1}^{\infty} \frac{1}{\sqrt{n_{i}^{1} ! n_{i}^{2} !}}\left(\frac{-1}{\sqrt{2}} D_{i}^{-}\right)^{n_{i}^{1}}\left(\frac{1}{\sqrt{2}} D_{i}^{+}\right)^{n_{i}^{2}} \\
& \times\left.\exp \left(\frac{-1}{2} \mathbf{v}^{T} \xi \mathbf{v}\right)\right|_{v=0} .
\end{aligned}
$$

The various quantities appearing in the above expression are given by

$$
\begin{aligned}
C(\lambda ; p)= & \exp \left(-\frac{1}{2} p^{2} \mathbf{k}^{T}(\mathbf{1}+\boldsymbol{\xi}) \mathbf{k}+p \boldsymbol{\lambda}^{T}\left(\mathbf{A}^{-}-\mathbf{A}^{+} \boldsymbol{\xi}\right) \mathbf{k}\right. \\
& \left.+\frac{1}{2} \boldsymbol{\lambda}^{T}\left(\mathbf{A}^{-}-\mathbf{A}^{+} \boldsymbol{\xi}\right) \mathbf{A}^{(+) T} \boldsymbol{\lambda}\right) .
\end{aligned}
$$


with

$$
\lambda_{i}=\left(\begin{array}{c}
\lambda_{i}^{\prime} \\
\lambda_{i}
\end{array}\right) ; \quad \tilde{\lambda}_{i}=\left(\begin{array}{c}
\tilde{\lambda}_{i} \\
\tilde{\lambda}_{i}^{\prime}
\end{array}\right) ; \quad \mathbf{k}_{i}=\frac{-1}{\pi \sqrt{2}}\left(\begin{array}{c}
0 \\
(2 i-1)^{-\frac{3}{2}}
\end{array}\right) \text {, }
$$

(lambdas with a prime are those that couple to the odd modes of the creation operators in equation (.9)) and $D_{i}^{ \pm}=\rho_{i} \pm \tilde{\lambda}_{i}+\frac{d}{d v_{i}}$, where $v$ is a dummy variable and $\rho$ is given by

$$
\rho_{i}^{T}=\left[\lambda^{T}\left(\mathbf{A}^{-}+\mathbf{A}^{+} \boldsymbol{\xi}\right)\right]_{i}-p\left[\mathbf{k}^{T}(\mathbf{1}+\boldsymbol{\xi})\right]_{i} .
$$

The coherent state matrix (.11) can be used to define the restricted vertices in the half-string language as

$$
V_{N}=\int d X_{I} \int_{D_{N}} \frac{d \lambda_{1} \cdots d \lambda_{N} d \phi_{1} \cdots d \phi_{N}}{S L(2 ; C)}\left(\Omega_{1} A_{1} \cdots \Omega_{N} A_{N}\right),
$$

where

$$
\Omega(\phi, \lambda)=e^{i \phi\left(L_{0}-\widetilde{L}_{0}\right)} e^{\lambda M} .
$$

The operator $M=L_{1}-L_{-1}+\widetilde{L}_{1}-\tilde{L}_{-1}$ is the element of the $S L(2 ; C)$ subalgebra of the Virasoro algebra that reparametrises $\sigma$ in such a way as to shrink the segment between $\sigma=\frac{\pi}{4}$ and $\sigma=\frac{3 \pi}{4}$, while expanding its complement[15]. Although this operator leaves the points $\sigma=0, \pi$ and $\frac{\pi}{2}$ invariant, one is free to move these points to any other position by a rigid rotation since, in order to build the field theory, these vertices still have to be multiplied by the projection operator $P$ that ensures that the boundary conditions are satisfied. $D_{N}$ represents the region in moduli space such that the restricted polyhedra are obtained. The relevant region of the moduli space has been determined in [16] for $N=4$. For $N=1,2$ and 3 no $M$ insertions are necessary.

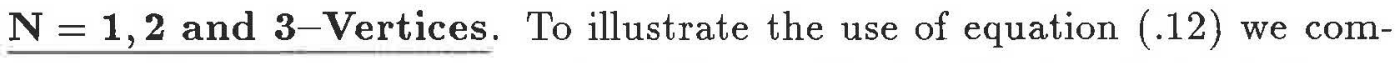
pute the 1-Vertex (Integration), the 2-Vertex (Sewing Ket) and the 3-Vertex (the $N$-Vertex, for any value of $\mathrm{N}$ with no operator insertions, can be easily obtained using the results of reference [14]). Setting $N=1$ in (.12) and using equation (.11), after a simple exercise in Gaussian integrals, we have

$$
I=\delta(P) \exp \left(\frac{1}{2}\left[\tilde{\lambda}^{2}-\lambda^{2}\right]\right)
$$

which is precisely the integration corresponding to folding the closed string along its diameter. 
Setting $N=2$ in equation (.12) and using once again (.11), we arrive at

$$
V_{2}=\delta\left(P_{1}+P_{2}\right) \exp \left({\widetilde{\lambda_{1}}}^{T} \cdot{\widetilde{\lambda_{2}}}_{-\lambda_{1}}{ }^{T} \cdot \lambda_{2}\right) .
$$

Thus reproducing the standard result.

Now that we have seen how the first two vertices are obtained in the halfstring formulation, we proceed to the computation of the 3-vertex. After performing the usual integration over $X_{I}$ in equation (.12), the part in the vertex involving the trace is given explicitly by

$$
\begin{aligned}
& \operatorname{Tr}\left(A_{1} \cdot A_{2} \cdot A_{3}\right)=\prod_{i=1}^{3} C\left(\lambda_{i} ; P_{i}\right) \sum_{\left\{n_{i}^{(r)}\right\}} \prod_{i=1}^{\infty} \frac{1}{n_{i}^{(1)} ! \cdot n_{i}^{(2)} ! \cdot n_{i}^{(3)} !} \\
& \times\left(-\frac{1}{2} D_{i}^{+(1)} D_{i}^{-(3)}\right)^{n_{i}^{(1)}}\left(-\frac{1}{2} D_{i}^{+(2)} D_{i}^{-(1)}\right)^{n_{i}^{(2)}}\left(-\frac{1}{2} D_{i}^{+(3)} D_{i}^{-(2)}\right)^{n_{i}^{(3)}} \\
& \times\left.\exp \left(-\frac{1}{2} \mathbf{v} \boldsymbol{\xi} \mathbf{v}\right)\right|_{\mathbf{v}=0} .
\end{aligned}
$$

This time we have embedded the above quantities in the vector space spanned by the three strings e.g., $\mathbf{v}=\left(\mathbf{v}^{1}, \mathrm{v}^{2}, \mathbf{v}^{3}\right)$, where the superindices 1,2 , and 3 refer the three strings 1,2 and 3 respectively. Performing the sum over $n_{i}^{(r)}$ and carrying out the derivatives in the auxiliary variable $\mathbf{v}$ using techniques in quadratic forms and after a tedious, although straightforward, calculation one ends up after diagonalizing etc. with

$$
\begin{aligned}
V_{3}=\delta\left(\sum_{r=1}^{3} P^{(r)}\right) & \exp \left(b \sum_{r=1}^{3} p^{(r)^{2}}\right) \exp \left(\overrightarrow{\tilde{\lambda}}^{T} \mathbf{E} \overrightarrow{\tilde{\lambda}}+\vec{\lambda}^{T} \mathbf{F} \vec{\lambda}\right) \\
& \times \exp \left(\overrightarrow{\tilde{\lambda}}^{T} \mathbf{G}^{\prime} \overrightarrow{\mathbf{P}}+\vec{\lambda}^{T} \mathbf{G} \overrightarrow{\mathbf{P}}+\vec{\lambda}^{T} \mathbf{H} \overrightarrow{\tilde{\lambda}}\right) .
\end{aligned}
$$

Where boldface quantities are vectors in the space spanned by the three strings, namely, $\overrightarrow{\mathbf{P}}=\left(P^{(1)} \mathbf{k}, P^{(2)} \mathbf{k}, P^{(3)} \mathbf{k}\right), \vec{\lambda}=\left(\boldsymbol{\lambda}^{(1)}, \lambda^{(2)}, \lambda^{(3)}\right)$, and $\overrightarrow{\tilde{\lambda}}^{\prime}=\left(\tilde{\boldsymbol{\lambda}}^{(1)}, \tilde{\boldsymbol{\lambda}}^{(2)}, \tilde{\boldsymbol{\lambda}}^{(3)}\right)$. The quantities $\mathbf{E}, \mathbf{F}, \mathbf{G}, \mathbf{G}^{\prime}$ and $\mathbf{H}$ are $3 \times 3$ dimensional matrices whose entries are infinite dimensional matrices. They are given explicitly by

$$
\begin{aligned}
b & =-\frac{1}{2} \overrightarrow{\mathbf{k}}^{T}\left(\mathbf{A}^{-\mathbf{T}}+\mathbf{A}^{+\mathbf{T}}\right)\left(2 \mathbf{A}^{-\mathbf{T}}+\mathbf{A}^{+\mathbf{T}}\right)^{-1} \overrightarrow{\mathbf{k}} \\
\mathbf{E}^{i, j} & =\frac{1}{2}\left(\delta_{i, j+1}+\delta_{i, j-1}\right) I-\cos \frac{2 \pi(i-j)}{3} \mathbf{A}^{+\mathbf{T}}\left(2 \mathbf{A}^{-\mathbf{T}}+\mathbf{A}^{+\mathbf{T}}\right)^{-1}, \\
\mathbf{F}^{i, j} & =\frac{1}{3}\left[(-)^{i+j}-1\right] I+
\end{aligned}
$$




$$
\begin{aligned}
& +\frac{2}{3} \cos \frac{2 \pi(i-j)}{3}\left(2 \mathbf{A}^{-\mathbf{T}}+\mathbf{A}^{+\mathbf{T}}\right)^{-1}\left(2 \mathbf{A}^{+\mathbf{T}}+\mathbf{A}^{-\mathbf{T}}\right), \\
\overrightarrow{\mathbf{G}}^{\prime i, j} & =\frac{2}{\sqrt{3}} \sin \frac{2 \pi(i-j)}{3}\left(2 \mathbf{A}^{-}+\mathbf{A}^{+}\right)^{-1}\left(\mathbf{A}^{+}+\mathbf{A}^{-}\right) \overrightarrow{\mathbf{k}} \\
\overrightarrow{\mathbf{G}}^{i, j} & =\frac{2}{3}\left(\mathbf{A}^{-}+\mathbf{A}^{+}\right) \overrightarrow{\mathbf{k}}+ \\
& +\frac{2}{3} \cos \frac{2 \pi(i-j)}{3}\left(2 \mathbf{A}^{-\mathbf{T}}+\mathbf{A}^{+\mathbf{T}}\right)^{-1} \overrightarrow{\mathbf{k}} \\
\mathbf{H}^{i, j} & =\frac{2}{\sqrt{3}} \sin \frac{2 \pi(i-j)}{3}\left(2 \mathbf{A}^{-\mathbf{T}}+\mathbf{A}^{+\mathbf{T}}\right)^{-1},
\end{aligned}
$$

In arriving at the above results we have made use of the properties of the matrix $\mathbf{A}^{ \pm}[17]$ and the symmetries of the trigonometric functions involved several times.

This gives the final form of the vertex. The important thing to notice at this point is that this result is given in terms of the change of representation matrices $\mathbf{A}^{ \pm}$, through the combination $\left(2 \mathbf{A}^{-\mathbf{T}}+\mathbf{A}^{+\mathbf{T}}\right)^{-1}$. Once the inverse of this matrix is obtained, we can directly identify the elements in the exponent of equation (.13) with the Fourier components of the Neumann function corresponding to the 3 -string contact interaction.

Recall that these matrices live in the two-dimensional space designated by the even-odd modes of the string. However due to the symmetries of our expressions we only need $\left(2 M^{-T}+M^{+T}\right)^{-1}$. This matrix, as well as its generalization, has already been discussed in [11]; it can be expressed in terms of the coefficients $U_{n}$ and $V_{n},(n, m \geq 1)$ obtained in the Taylor expansion of the functions $\left(\frac{1+x}{1-x}\right)^{\frac{1}{3}}$ and $\left(\frac{1+x}{1-x}\right)^{\frac{2}{3}}$ respectively. One can check that

$$
\begin{aligned}
\left(2 M^{-T}+M^{+T}\right)_{n, m}^{-1}= & -\frac{[(2 n)(2 m-1)]^{1 / 2}}{2 \sqrt{3}} \\
& \times\left[\frac{V_{2 m-1} U_{2 n}+U_{2 m-1} V_{2 n}}{2 n-2 m+1}+\frac{U_{2 m-1} V_{2 n}-U_{2 n} V_{2 m-1}}{2 n+2 m-1}\right] .
\end{aligned}
$$

Hence for example $b=\frac{1}{8} \ln \frac{3^{3}}{2^{4}}=\frac{1}{4} N_{00}, \frac{1}{2} N_{00}$ is the Neumann coefficient coupling three open string tachyons [11]. This result is clearly consistent with the fact that for closed strings $P^{2}=\frac{1}{4} P_{R}^{2}+\frac{1}{4} P_{L}^{2}=\frac{1}{2} P_{\text {open }}^{2}$.

The rest of the coefficients

$$
\mathbf{R}_{m, n}=\left(\begin{array}{cc}
R_{m, n}^{(1)} & 0 \\
0 & R_{m, n}^{(2)}
\end{array}\right),
$$

where $\mathbf{R}$ stands for either $\mathbf{E}, \mathbf{F}, \mathbf{G}, \mathbf{G}^{\prime}$ or $\mathbf{H}$ (see equation (.7)) are given in ref. $[19]$. 
Notice that these coefficients are very closely related to the standard Neumann coefficients for the 3 open strings vertex [11].

To address the problem of mixing between left- and right-movers, it is necessary to express the above vertex in terms of the left- and right-moving standard oscillators $\alpha_{n}^{\dagger}$ and $\bar{\alpha}_{n}^{\dagger}$. This can be done in a straightforward way, using the results of ref. [19], we arrive at

$$
\begin{aligned}
& \mid V_{3}>=e^{\frac{1}{8} \ln \frac{3^{3}}{2^{4}}\left(\sum_{r=1}^{3} P_{\tau}^{2}\right)} \\
& \exp \frac{1}{\sqrt{2}}\left(\frac{\alpha_{-2 n}^{(i)}}{\sqrt{2 n}} \vec{G}_{n}^{(2) i, j} P^{j}-i \frac{\alpha_{-2 n+1}^{(i)}}{\sqrt{2 n-1}} \vec{G}_{n}^{(2) \prime i, j} P^{j}+\frac{\alpha_{-2 n}^{(i)}}{\sqrt{2 n}}\left(2 F_{n m}^{(2) i, j}\right) \frac{\alpha_{-2 m}^{(j)}}{\sqrt{2 m}}+\right. \\
& \left.-\frac{\alpha_{-2 n+1}^{(i)}}{\sqrt{2 n-1}}\left(2 E_{n m}^{(2) i, j}\right) \frac{\alpha_{-2 m+1}^{(j)}}{\sqrt{2 m-1}}-i \frac{\alpha_{-2 n}^{(i)}}{\sqrt{2 n}}\left(2 H_{n m}^{(2) i, j}\right) \frac{\alpha_{-2 m+1}^{(j)}}{\sqrt{2 m-1}}\right) \times \\
& \exp \frac{1}{\sqrt{2}}\left(\frac{\tilde{\alpha}_{-2 n}^{(i)}}{\sqrt{2 n}} \vec{G}_{n}^{(2) i, j} P^{j}+i \frac{\tilde{\alpha}_{-2 n+1}^{(i)}}{\sqrt{2 n-1}} \vec{G}_{n}^{(2) \prime i, j} P^{j}+\frac{\tilde{\alpha}_{-2 n}^{(i)}}{\sqrt{2 n}}\left(2 F_{r m}^{(2) i, j}\right) \frac{\tilde{\alpha}_{-2 m}^{(j)}}{\sqrt{2 m}}\right. \\
& \left.-\frac{\tilde{\alpha}_{-2 n+1}^{(i)}}{\sqrt{2 n-1}}\left(2 E_{n m}^{(2) i, j}\right) \frac{\tilde{\alpha}_{-2 m+1}^{(j)}}{\sqrt{2 m-1}}+i \frac{\tilde{\alpha}_{-2 n}^{(i)}}{\sqrt{2 n}}\left(2 H_{n m}^{(2) i, j}\right) \frac{\tilde{\alpha}_{-2 m+1}^{(j)}}{\sqrt{2 m-1}}\right) \times \\
& \exp \frac{1}{\sqrt{2}}\left(\frac{\alpha_{-2 n}^{(i)}}{\sqrt{2 n}}\left(E_{n m}^{(1) i, j}+E_{m n}^{(1) j, i}+F_{n m}^{(2) i, j}+F_{m n}^{(2) j, i}\right) \frac{\tilde{\alpha}_{-2 m}^{(j)}}{\sqrt{2 m}}\right. \\
& +\frac{\alpha_{-2 n+1}^{(i)}}{\sqrt{2 n-1}}\left(E_{n m}^{(2) i, j}+E_{m n}^{(2) j, i}+F_{n m}^{(1) i, j}+F_{m n}^{(1) j, i}\right) \frac{\tilde{\alpha}_{-2 m+1}^{(j)}}{\sqrt{2 m-1}} \\
& +i \frac{\alpha_{-2 n}^{(i)}}{\sqrt{2 n}}\left(-H_{n m}^{(2) i, j}+H_{m n}^{(1) j, i}\right) \frac{\tilde{\alpha}_{-2 m+1}^{(j)}}{\sqrt{2 m-1}} \\
& \left.-i \frac{\tilde{\alpha}_{-2 n}^{(i)}}{\sqrt{2 n}}\left(-H_{n m}^{(2) i, j}+H_{m n}^{(1) j, i}\right) \frac{\alpha_{-2 m+1}^{(j)}}{\sqrt{2 m-1}}\right)\left|0>_{1}\right| 0>_{2} \mid 0>_{3}
\end{aligned}
$$

here $\mid 0>_{i}, i=1,2,3$ are the vacua corresponding to strings 1,2 and 3 respectively.

Using ref. [19], it is easy to see that, in fact, there is no mixing between right and left moving oscillators in the above equation. For example for the odd-even modes, the mixing term is proportional to $H^{(1)}-H^{(2) T}=0$.

Notice that we started with a vertex, with no pre-assumption about non mixing between right-moving factors and left-moving factors. The fact that no mixing between left- and right-movers appears in the final expression of the vertex seems to suggest that factorization is not special to tachyons but it is a generic feature. In fact we have checked that this property of $V_{N}$ holds for any $\mathrm{N}$. Therefore we conclude that factorization is indeed a general rule. 
This vertex has the general form:

$$
\exp \frac{1}{2}\left(\alpha_{-n}^{r} N_{n m}^{r s} \alpha_{-m}^{s}+\tilde{\alpha}_{-n}^{r} N_{n m}^{r s *} \tilde{\alpha}_{-m}^{s}\right)\left|0>_{1}\right| 0>_{2} \mid 0>_{3},
$$

where $N_{n m}^{r s *}$ denotes the complex conjugate of $N_{n m}^{r s}$.

This is just what one would expect from the standard perturbative treatment of closed strings. We have also calculated several scattering amplitudes (see ref. [19]) in our formulation and found that they do agree with the standard results found in the literature. For example, we obtain for the Tachyon - Tachyon Graviton scattering amplitude

$$
A_{3}(T, T, G)=\frac{1}{4}\left(\frac{P_{1}}{2}-\frac{P_{2}}{2}\right)^{\mu}\left(\frac{P_{1}}{2}-\frac{P_{2}}{2}\right)^{\nu} G_{\mu, \nu}\left(P_{3}\right)
$$

This should be compared with the standard result

$$
A^{\text {closed }}\left(P_{3}\right)=A^{\text {open }}\left(P_{3} / 2\right) A^{\text {open }}\left(P_{3} / 2\right)^{*}
$$

Where $A^{\text {open }}\left(P_{3}\right)$ is the open string Tachyon - Tachyon - Vector scattering amplitude which is given by

$$
A^{\text {open }}\left(P_{3}\right)=\frac{1}{2}\left(P_{1}-P_{2}\right)^{\mu} A_{\mu}\left(P_{3}\right),
$$

here $P_{1}$ and $P_{2}$ are the momenta of the tachyons and $A_{\mu}\left(P_{3}\right)$ represents the vector particle (one has to make the identification $G_{\mu, \nu} \equiv A_{\mu} A_{\nu}$ at the end.).

Conclusions. We have showed that closed-string fields can be represented by infinite dimensional matrices in the Half-String Language. The ghost sector of the theory can also be treated within the half-string formulation in a similar way as in references $[12,13]$. We have constructed explicitly the vertices $V_{N}$ for $N=1,2$ and 3 . We obtained the correct vertex that couples three strings together. The vertices that we have obtained can be seen to satisfy the "general gluing and resmoothing theorem" of [18] in an almost trivial way [19].

The important point to notice here is that we have made no pre-assumption about factorization and found out that left- and-right moving oscillators, which are mixed to start with in the interaction, decouple, in our formalism, at the end of the calculation in the vertices (this holds for higher order vertices as well). This is in accordance with the so long made"assumption" about factorization. The absence of a mixing term is not only reassuring, but, as mentioned before, it is the first rigorous proof of the assumption that the left- and right-oscillators do indeed decouple at the level of vertices (the equivalent statement at the 
level of perturbative closed-string theory would be that the vertex operators are simply the product of two open-string vertex operators). Furthermore, this result seems to be essential for a consistent string field theory of Heterotic strings to be possible.

The work of A.A. has been supported by the SSR, Tripoli, Libya. The work of J.B. has been supported by CICYT under grant number 93-0234.

\section{References}

[1] E. Witten, Nucl. Phys. B268, 253 (1986).

[2] S. B. Giddins, E. Martinec, Nucl.Phys. B278, 91 (1986).

[3] M. Kaku, Phys. Rev.D38, 3052 (1988) and M. Kaku and J. Lykken, Phys. Rev.D38, 3067 (1988).

[4] B. Zwiebach, Ann. Phys. 186, 111 (1988).

[5] B. Zwiebach, Nucl. Phys. B336, 185 (1990).

[6] M. Saadi and B. Zwiebach, Annals of Phys 192, 213 (1989).

[7] T. Kugo, H. Kunitomo and K. Suehiro, Phys. Lett. B226, 48 (1989).

[8] T. Kugo and K. Suehiro, Nucl. Phys. B337, 434 (1990).

[9] H.M. Chan and S.T. Tsou, Phys. Rev. D35, 2474 (1987).

[10] H.M. Chan and S.T. Tsou, Phys. Rev. D39, 555 (1989).

[11] J. Bordes, H.M. Chan, L. Nellen and S.T. Tsou, Nucl.Phys. B351, 441 (1991) .

[12] A. Abdurrahman, F. Anton and J. Bordes, Nucl. Phys. B397, 260 (1993).

[13] A. Abdurrahman, F. Anton and J. Bordes, Nucl. Phys. B411, 693 (1994).

[14] J. Bordes, A. Abdurrahman and F. Anton, Phys. Rev. D49, 2966 (1994).

[15] H.M. Chan, J. Bordes, S.T. Tsou, and L. Nellen, Phys. Rev. D44, 1786 (1991).

[16] F. Anton, A. Abdurrahman and J. Bordes, Phys. Lett. B 327, 234 (1994). 
[17] F. Anton, D.Phil. Thesis, Department of Theoretical Physics, Oxford University, 1993.

[18] A. LeClair, M.E. Peskin and C.R. Preitschopf, Nucl. Phys. B317, 411 (1989).

[19] F. Anton, A. Abdurrahman and J. Bordes, Rutherford Appleton Laboratory preprint RAL-94-065 

